\title{
Determination of water content in corn stover silage using near-infrared spectroscopy
}

\author{
Maoqun Zhang ${ }^{1,2}$, Chao Zhao ${ }^{1 *}$, Qianjun Shao ${ }^{3}$, Zidong Yang ${ }^{1}$, Xuefen Zhang ${ }^{1}$, \\ Xiaofeng $\mathrm{Xu}^{1}$, Muhammad Hassan ${ }^{4}$ \\ (1. National Engineering Research Center for Wood-based Resource Utilization, School of Engineering, Zhejiang A\&F University, \\ Lin'an, Zhejiang 311300, China; 2. Zhejiang Collaborative Center of Efficient Utilization of Bamboo Resources, Zhejiang 311300, China; \\ 3. Faculty of Mechanical Engineering \& Mechanics, Ningbo University, Ningbo, Zhejiang 315211, China; \\ 4. US-Pakistan Centre for Advanced Studies in Energy, National University of Science and Technology, Islamabad 44000, Pakistan)
}

\begin{abstract}
The aim of this study was to evaluate the feasibility of utilizing near-infrared spectroscopy to determine the water content of corn stover silage across a wide range. The water contents of 208 samples were measured, and their corresponding near-infrared spectra were simultaneously collected. The effects of different preprocessing methods, such as derivation, standard normal variety (SNV), multiplicative scatter correction (MSC), and non-preprocessing methods for the obtained near-infrared spectra on the performance of calibration models were compared. The calibration models were established by modified partial least squares (MPLS) regression. The results showed that the calibration model developed from the successive preprocessing of MSC and first-order derivation (1-D) achieved the optimal performance. The correlation coefficients of the calibration and validation subset were 0.974 and 0.949 , respectively, and the standard errors of the calibration and cross validation were $4.249 \%$ and $4.256 \%$, respectively. External validation was performed on 60 samples. The correlation coefficient between the measured and predicted values of the calibration model was 0.973 and the prediction model's relative percent deviation was 4.317. This indicated that the mathematical model of near-infrared spectroscopy predicted the water content in corn stover silage with high accuracy. The study showed that the near-infrared spectroscopy technology can be used for rapid and non-destructive testing across a wide range of water contents in the corn stover silage.
\end{abstract}

Keywords: near-infrared spectroscopy, water, non-destructive measurement, corn stover silage DOI: $10.25165 /$ j.ijabe.20191206.4914

Citation: Zhang M Q, Zhao C, Shao Q J, Yang Z D, Zhang X F, Xu X F, et al. Determination of water content in corn stover silage using near-infrared spectroscopy. Int J Agric \& Biol Eng, 2019; 12(6): 143-148.

\section{Introduction}

Corn (Zea mays L.) is one of the high and stable-yield crops in the world, and corn stover is one of the three major agricultural residues in China which accounts for $36.81 \%$ of China's total straw production $^{[1,2]}$. Although still contains rich nutrients suitable for livestock, corn stover is usually incinerated or ploughed back in the soil. Silage is a common preserved feed in many countries, and corn stover silage is mainly used as animal forage $\mathrm{e}^{[3]}$. At present, many dairy farms adopt the corn stover silage to raise cows, so as to ensure the stable quality of roughage in the diet structure and improve the milk yield. Therefore, the ensiling of fresh corn stover is an attractive raw material for forage production due to its high content of carbohydrates and easy degradability. Water,

Received date: 2019-01-14 Accepted date: 2019-10-21

Biographies: Maoqun Zhang, Master candidate, research interests: near-infrared spectroscopy and its application, Email: 2465144243@qq.com; Qianjun Shao, PhD, Professor, research interests: biomass property detection, Email: shaoqianjun@nbu.edu.cn; Zidong Yang, PhD, Professor, research interests: nondestructive detection, Email: yzd66@126.com; Xuefen Zhang, Master, research interests: biomass property detection, Email: 404365047@, qq.com; Xiaofeng $\mathbf{X u}, \mathrm{PhD}$ candidate, research interests: biomass property detection, Email: penguinuaa@sina.com; Muhammad Hassan, PhD, research interests: biomass and bioenergy, Email: engrhasan74@yahoo.com.

*Corresponding author: Chao Zhao, PhD, Associate Professor, research interests: biomass property detection. Mailing address: 666\# Wusu street, Lin'an district, Hangzhou 311300, Zhejiang, China. Tel: +86-571-63746877, Email: zhaochao@zafu.edu.cn. temperature, and lactic acid bacteria have great influence on the quality of forage during ensiling process ${ }^{[4]}$. Of which, the water content of the corn stover silage is the most fundamental and important parameter ${ }^{[5,6]}$. For ensiling process, it is very important to realize the fast determination of water content, and then adjusting it for better forage quality. However, the conventional chemical analysis method for determining water content is time-consuming and labor-intensive $e^{[7,8]}$ and could not meet the requirements of online analysis. Therefore, research on rapid and non-destructive testing of water content in corn stover silage could improve the detection efficiency, reduce the analysis cost, provide an efficient analysis platform, and information on straw utilization $^{[9,10]}$.

Generally, near-infrared spectroscopy provides the vibrational information of $\mathrm{O}-\mathrm{H}, \mathrm{C}-\mathrm{O}$, and other groups inside a substance. When combined with chemometric methods, near-infrared spectroscopy could be used to perform qualitative or quantitative analysis of the groups mentioned above $\mathrm{e}^{[11-14]}$. As a new and non-destructive testing technology, near-infrared spectroscopy has been widely utilized in agriculture, pharmacy, and chemical industry ${ }^{[15-17]}$. In recent years, advanced non-destructive testing techniques, such as terahertz spectroscopy ${ }^{[18]}$ and laser-induced breakdown spectroscopy ${ }^{[19]}$, have appeared. Multi-component parameters in fruits, grains and food could be determined by near-infrared spectral information, especially the rapid and non-destructive detection of water content. Extensive studies on straw analysis have been conducted using near-infrared 
spectroscopy. Huang et al. ${ }^{[20,21]}$ successfully applied this technology to determine of the water content and calorific value of rice straw and wheat straw. Liu et al. ${ }^{[22]}$ established prediction models for water and ash content of corn stover using near-infrared spectroscopy. Wu et al. ${ }^{[23]}$ analyzed the cellulose content of corn stover using near-infrared spectroscopy. Some researchers constructed an analytical model to analyze the feed quality of corn stover, such as dry matter digestibility, acid detergent fiber, neutral detergent fiber and soluble sugars ${ }^{[24,25]}$. Even the decomposition of corn stover has been studied using near-infrared spectroscopy ${ }^{[26]}$. To the best of our knowledge, the detection of water content using near-infrared spectroscopy for corn stvoer silage has rarely been reported.

Many researchers have claimed that the changes in sample composition affect the accuracy of near-infrared calibrations ${ }^{[11]}$. Investigations by Huang et al. ${ }^{[20]}$ suggested that the variations in moisture and calorific value of wheat straw had a significant influence on the prediction of the calibration model. To solve this wide range of testing parameter, they divided the test samples into three subsets according to the chemical values (labeled as high, middle and low) using a local algorithm. A calibration model for the rapid determination of neutral detergent fiber (NDF) and acid detergent fiber (ADF) in corn stover silage was obtained by near-infrared spectroscopy in a recent study, in which the NDF and ADF ranges $43.39 \%-84.56 \%$ and $25.61 \%-57.13 \%$, respectively ${ }^{[25]}$. The wide variations in NDF and ADF were caused by the different varieties, different growth stages, and different parts of the samples. This indicates that a parameter with a wide range could also be predicted using near-infrared spectroscopy. Furthermore, the reported studies had mainly focused on the quality of straw feed in terms of cellulose content, calorific value, ash content, and even decomposition process, where the detection of water is almost based on the safe water content of straw, in which the highest water content of straw is $20.44 \%{ }^{[20]}$. To the best of our knowledge, the detection of water content higher than $70 \%$ using near-infrared spectroscopy for straw has rarely been reported.

In this study, corn stover silage with a wide-range of water content $(9.82 \%-71.09 \%)$ was used as the research object and near-infrared spectroscopy was utilized to determine the water content of this silage. A prediction model for water content in the corn stover silage was established and the validation of the model was evaluated.

\section{Materials and methods}

\subsection{Sample collection and preparation}

The corn stover silage was harvested in July 2012 from Quanjiao Yangzhuang Farm $\left(32.10^{\circ} \mathrm{N} ; 118.23^{\circ} \mathrm{E}\right)$, Anhui Province, China. The corn variety was Xin'an $5 \#$, and it took about $85 \mathrm{~d}$ from sowing to harvesting. After cutting the upper corn ear and lower root, the corn stalk and leaf were used as corn stover silage. These raw materials were then cut into strips of about 5-10 mm, evenly mixed, and placed in a laboratory environment for water analysis and spectral acquisition.

\subsection{Chemical analysis}

The water content of corn stover silage was determined based on wet basis in this study. Only one water content value of the initial corn stover silage could be obtained. To obtain a wide range of water content values of the experimental materials, the initial corn stover silage was put in an electric blast drying oven (DHG series 9075A, Shanghai Yiheng Scientific Instrument Co., Ltd. China) and a sample was taken at 10-min intervals until the silage was dried below the safe water content. The near-infrared spectrum was recorded, and water content measured for each sample. The near-infrared spectrum of each sample was recorded as described in Section 2.3. Next, the sample were marked and filled into 3 aluminum boxes (diameter $=80 \mathrm{~cm}$, height $=50 \mathrm{~cm}$, weight $=15 \mathrm{~g}$ ) to measure their water contents, and the average value was recorded as the water content of the sample. Each aluminum box was randomly filled with corn stover silage. The initial weight of each corn stover silage sample varied from $3.6 \mathrm{~g}$ to $12.1 \mathrm{~g}$ depending on the water content. The water content was determined on a wet basis according to GB/T 14095-2007 ${ }^{[27]}$. Samples were dried to a constant quality (without quality change), and then their water contents were calculated. This ensured that the spectral information corresponded to their water content. Overall, 208 samples, with water contents ranging from $9.82 \%$ to $71.09 \%$, were obtained in this experiment.

\subsection{Collection of near-infrared spectra}

The near-infrared spectra of the samples were obtained on a near-infrared spectrometer (Infraxact Lab, Foss Instruments, Denmark) that used a monochromator-based NIR reflectance and transflectance analyzer. The spectral scanning range was $570-1850 \mathrm{~nm}$, with increments of $2 \mathrm{~nm}$, and the data acquisition frequency was 3 s. Figure 1 shows the schematic of the near-infrared spectra collection process of corn stover silage. The light source from a tungsten halogen lamp was dispersed by a holographic grating and then split into single-wavelength light. The dispersed light entered the sample and interacted with the sample molecules. Si (570-1100 nm) and InGaAs (1100-1850 nm) detectors collected the spectra in the NIR region. The spectral acquisition and storage were performed by using ISI scan operating software. The samples were scanned by a small sample cup arrangement in the reflectance mode. During the spectral acquisition process, the ambient temperature was maintained at $26^{\circ} \mathrm{C}$. The bandwidth accuracy was less than $0.1 \mathrm{~nm}$ and the exposure time was $400 \mathrm{~ms}$. Each sample was registered three times and scanned separately, and the average value was recorded as the near-infrared spectrum of the sample ${ }^{[5]}$.

\subsection{Establishment of near-infrared calibration model}

To establish the calibration model, the measured water content values of the samples were input into the modeling program. Notably, each measured water content was related to its corresponding scanning spectrum. Samples of 208 were randomly divided into a calibration set and a cross validation set in a ratio of $3: 1$, and the calibration and validation sets were used for model establishment and reliability evaluation, respectively.

When establishing the calibration model, the obtained spectrum was first smoothed by four data interval window points (every $8 \mathrm{~nm}$ ) according to GB/T 18868-2002 to eliminate the influences of noise ${ }^{[28]}$. Then, different spectral preprocessing methods were tested to reduce unwanted variation due to sources not related to water properties. The preprocessing methods were first-order derivation (1-D), second-order derivation (2-D), standard normal variation (SNV), multiplicative scatter correction (MSC), and the combination of the above mentioned methods ${ }^{[5]}$. According to a previous study, smoothing mainly removes the interference of high-frequency noise on the signal. Derivation could effectively eliminate background-induced interference and baseline drift or rotation. SNV eliminates the effects of the changes due to the optical-path or dilution. MSC could eliminate the spectral baseline shift due to the difference in size and shape of samples $^{[15,16]}$ 


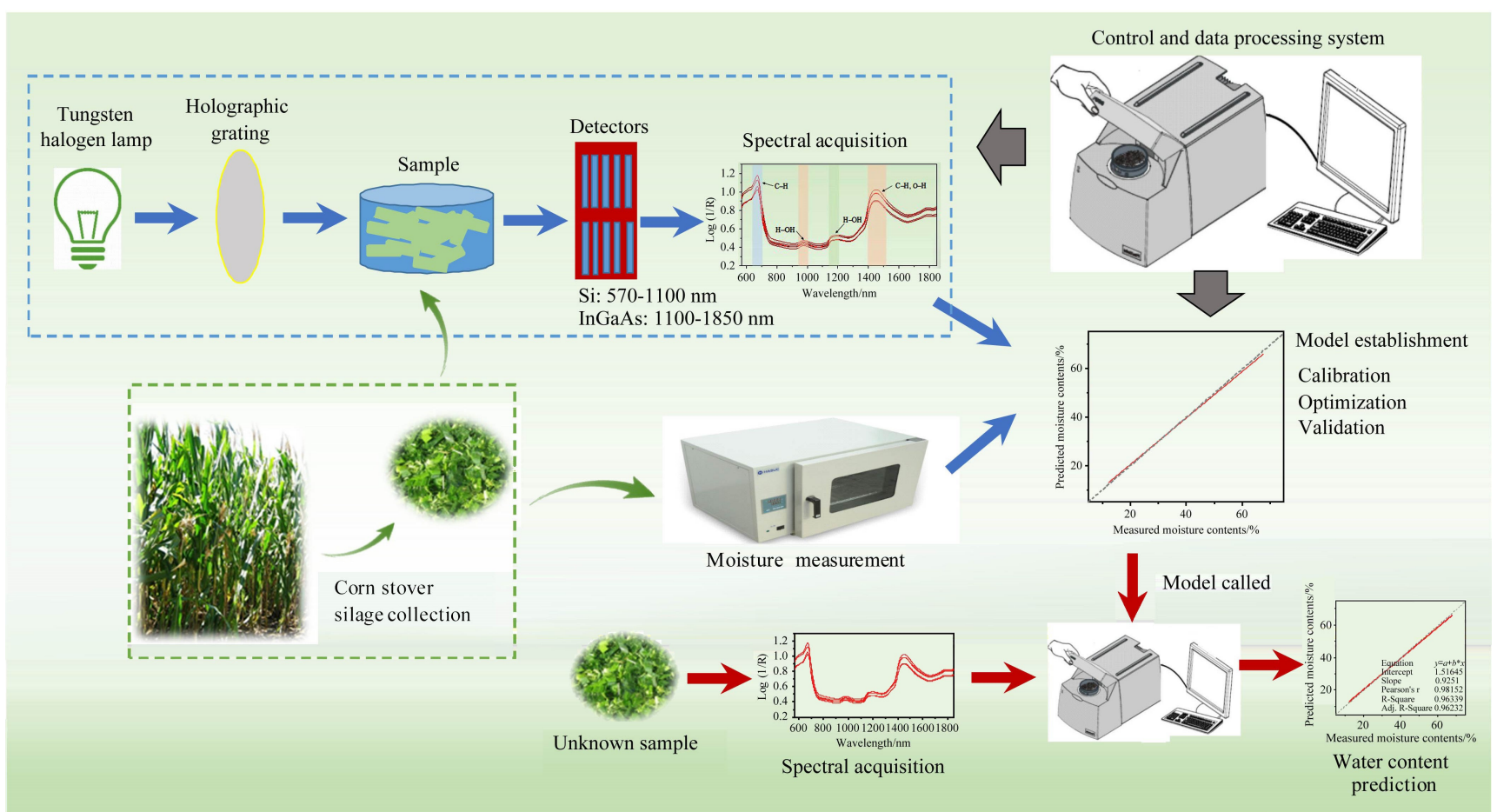

Figure 1 Schematic of collection process of near-infrared spectra for corn stover silage

The calibration model was established by the modified partial least squares (MPLS) method. The optimal factor number adopted by the model was determined by a cross validation set, including root mean square error of calibration (RMSEC), calibration correlation coefficient $(R)$, root mean square error of cross validation (RMSECV) and cross validation correlation coefficient $\left(R_{C V}\right)$. The optimal model was usually with good enough compromise between low RMSEC and RMSECV, and high $R$ and $R_{C V}^{[9]}$.

RMSEC, RMSECV and the root mean square error of prediction (RMSEP) were calculated as follows ${ }^{[5]}$ :

$$
\begin{aligned}
& \text { RMSEC }=\sqrt{\frac{\sum_{i=1}^{I}\left(m_{i}-\bar{m}_{i}\right)^{2}}{I-1}} \\
& \text { RMSECV }=\sqrt{\frac{\sum_{i=1}^{I_{l}}\left(m_{i}-\bar{m}_{i}\right)^{2}}{I_{l}}} \\
& \operatorname{RMSEP}=\sqrt{\frac{\sum_{i=1}^{I_{t}}\left(m_{i}-\bar{m}_{i}\right)^{2}}{I_{t}}}
\end{aligned}
$$

where, $m_{i}$ is the water content of sample $i, \% ; \bar{m}_{i}$ is the water content of model prediction for sample $i, \% ; I$ is the number of calibration samples; $I_{l}$ is the number of samples in the cross validation set; and $I_{t}$ is the number of prediction samples.

\subsection{Evaluation of the calibration model}

To examine the accuracy of the model, a cross verification set was performed. The accuracy of the model's prediction was judged by comparing the difference between the predicted and the measured water content values. The accuracy of the model was evaluated by the ratio of the performance to deviation (RPD). If $R P D \geq 3$, the established calibration model could be well fitted to the measured results. If $2.5<R P D<3$, the prediction accuracy needs to be further improved, and $R P D \leq 2.5$, indicates that it is difficult to predict the quantitative analysis of the established calibration model ${ }^{[5,20]}$. It should be noted that it was necessary to expand the coverage of the calibration samples when the water content to be determined exceeded the range of the existing calibration model. RPD was calculated as follows:

$$
R P D=\frac{S D}{R M S E P}
$$

where, $S D$ is the standard deviation of the validation set.

\section{Results and Discussion}

\subsection{Analysis of the water content measurement results}

Table 1 shows that the water contents of the 208 samples ranged from $9.82 \%$ to $71.09 \%$, much higher than the coverage of water content in the general near-infrared spectrum water detection calibration set. Liu et al. reported that the water content of corn stover ranged from $3.15 \%$ to $6.75 \%{ }^{[22]}$, while Huang et al. reported the water content of wheat/rice straw to range from $5.13 \%$ to $20.44 \%{ }^{[21]}$ and the water content of soybean was predicted to range from $6.92 \%$ to $13.71 \%{ }^{[9]}$. Water contents in these studies varied by less than $15 \%$. In an interesting study, water uptake while barley steeping was monitored the by near-infrared spectroscopy over a large scale range of water content $(28.5 \%-97.9 \%)^{[29]}$. The samples were randomly divided into a calibration set and a verification set in a 3:1 ratio. The results of the sample partitioning are also listed in Table 1 . These results suggest that the water content ranges of the calibration and the verification sets were both widely distributed and were consistent with the overall samples. The water content range of verification set was in that of calibration set.

Table 1 Water content measurement results and sample set partition

\begin{tabular}{cccccc}
\hline Sample Set & $\begin{array}{c}\text { Sample } \\
\text { Number }\end{array}$ & Max/\% & Min/\% & $\begin{array}{c}\text { Average } \\
/ \%\end{array}$ & $\begin{array}{c}\text { Standard } \\
\text { Deviation }\end{array}$ \\
\hline Total & 208 & 71.09 & 9.82 & 38.28 & 18.67 \\
Calibration set & 156 & 71.09 & 9.82 & 38.80 & 18.84 \\
Validation set & 52 & 67.64 & 12.11 & 36.72 & 17.97 \\
\hline
\end{tabular}

\subsection{Characteristics of near-infrared spectra}

Figure 2 shows the near-infrared spectra of 5 corn stover silage samples. It was observed that different samples exhibited almost similar spectral morphology over the scanned wavelength range, 
and this was similar to previous reported observations ${ }^{[9,30]}$. There were multiple absorption peaks in the spectra over the entire wavelength range, which reflected the composition of water and organic matter (mainly fibrous matter) in the silage. The spectra showed strong absorption peaks around $670 \mathrm{~nm}$ and $1450 \mathrm{~nm}$, and the absorption peaks also appeared at around $970 \mathrm{~nm}$ and $1190 \mathrm{~nm}$. According to previous studies, the strongest absorption peak at around $670 \mathrm{~nm}$ was due to the out-of-plane bending vibration of the $\mathrm{C}-\mathrm{H}$ bond associated with cis olefin ${ }^{[15]}$. The absorption peaks around $1450 \mathrm{~nm}$ are associated with either $\mathrm{C}-\mathrm{H}$ combinations (aromatic groups) or the $\mathrm{O}-\mathrm{H}$ first overtone (water) ${ }^{[29]}$. The absorption peaks around $970 \mathrm{~nm}$ and $1190 \mathrm{~nm}$ are attributed to the hydroxyl stretching vibration of either water molecule or the $\mathrm{H}-\mathrm{OH}$ variable angle vibration. These results are in agreement with previous studies. Many researchers have reported that the typical water regions in near-infrared spectra are at 1351-1667 nm and $1852-2128 \mathrm{~nm}^{[15,23]}$. Therefore, the near-infrared spectra obtained herein covered the multiplier and frequency spectrum band of water molecule vibration. All the above-mentioned findings indicated that the spectra of the samples could provide the information necessary for determination of water content.

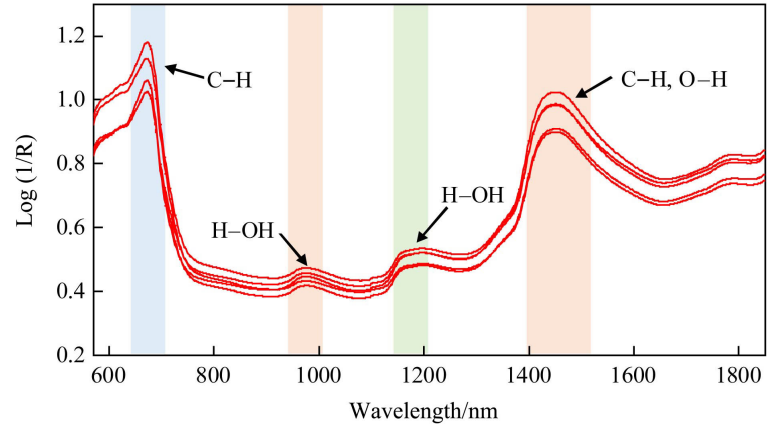

Figure 2 Near-infrared spectra of corn stover silage samples

3.3 Establishment and optimization of near-infrared calibration model

The quantitative relationship between the spectra and water content for each sample was established by using the MPLS method in the whole spectra. When establishing the calibration model, the obtained spectra were first smoothed by four data interval window points (every $8 \mathrm{~nm}$ ) according to $\mathrm{GB} / \mathrm{T}$ $18868-2002^{[28]}$. Signal smoothing was one of the most common ways to eliminate the interference of high-frequency noise. Then, processing was performed by first order derivation (1-D), second order derivation (2-D), standard normal variate (SNV) and multiplicative scatter correction (MSC). The calibration models established from the four different preprocessing methods are shown in Table 2. Compared with the modeling results of the raw spectra, the model established by the SNV method had the worst parameters of the calibration and the verification sets, while the other preprocessing methods were found to significantly improve the stability and predictability of the model. This indicated that there was almost no optical path change in spectral response. The correlation coefficients of both the calibration and verification sets based on derivation (1-D and 2-D) preprocessing were above 0.9, indicating that derivation could effectively eliminate background-induced interference and baseline drift, and it had a strong characteristic extraction ability from near-infrared spectra. However, the RMSECV of the verification set based on the 2-D preprocessing method was slightly higher than that based on the 1-D preprocessing method, whereas the $\mathrm{R}_{\mathrm{CV}}$ of verification set based on the 2-D preprocessing method was slightly lower than that based on the 1-D preprocessing method. This suggested that second order derivation caused the noise to be over-amplified, and hence, the model performance was degraded. The $\mathrm{R}_{\mathrm{CV}}$ of the verification set based on the MSC preprocessing method was 0.936 , which indicated that the surface scattering characteristics of the sample had significant influence on the spectrum. This may be due to the different size and shape of the corn stalk and leaf in the sample. In summary, there was almost no optical path change in spectral response, while surface scattering characteristics of the sample greatly influenced on the spectra under the described experimental conditions. Derivation had strong characteristic extraction ability from the near-infrared spectra. Different pretreatment methods were utilized to eliminate noise and interference from the spectra. According to reported studies, some spectra only need derivation (either the first or the second order) ${ }^{[29]}$, but some spectra need additional processing methods, such as either SNV or MSC which are closely related to the experimental environment and the tested object ${ }^{[20,21,24]}$.

Table 2 Results of MPLS models for water content based on the spectra of corn stover silage

\begin{tabular}{cccccc}
\hline \multirow{2}{*}{$\begin{array}{c}\text { Processing } \\
\text { method }\end{array}$} & \multicolumn{2}{c}{ Calibration set } & & \multicolumn{2}{c}{ Validation set } \\
\cline { 2 - 3 } \cline { 5 - 5 } & $R M S E C / \%$ & $R$ & & $R M S E C V / \%$ & $R_{C V}$ \\
\hline Raw spectra & 10.265 & 0.838 & 10.316 & 0.698 \\
1-D & 5.617 & 0.955 & 5.637 & 0.911 \\
2-D & 5.679 & 0.953 & 5.719 & 0.909 \\
SNV & 12.465 & 0.759 & 12.474 & 0.573 \\
MSC & 4.762 & 0.968 & 4.780 & 0.936 \\
MSC+1-D & 4.249 & 0.974 & 4.256 & 0.949
\end{tabular}

Note: 1-D: the first-order derivation; 2-D: the second-order derivation; SNV: standard normal variate; MSC: multiplicative scatter correction.

According to the results of Table 2, it concluded that MSC and derivation had strong characteristic extraction ability from near-infrared spectra. To obtain a more accurate calibration model, a combination of MSC and 1-D preprocessing was performed. The results showed that the calibration model derived from the combined method gave the lowest RMSECV (4.256\%) and the highest $R_{C V}(0.949)$, thus demonstrating the best performance. That's to say, the optimal calibration model for determination of water content after selecting from different preprocessing methods, was obtained by successively preprocessing using MSC and 1-D. The experimental result was consistent with that of the optimal calibration model for predicting acid ADF content of corn stover silage over a wide range $(25.61 \%-57.13 \%)^{[25]}$. According to published reports, almost all the optimal calibration models were obtained by the combined preprocessing of the spectrum. Huang et al. suggested that the optimal calibration models for moisture content and calorific value of corn stover were adopted from combined SNV and derivation preprocessing ${ }^{[21]}$. The optimal calibration models for determining feed quality of corn stover were adopted from combined derivation and MSC preprocessing ${ }^{[24]}$, and that for composition of corn stover was adopted from the combination of derivation and Karl Norris filter preprocessing ${ }^{[22]}$.

\subsection{External validation of the calibration model}

An external verification set was performed to examine the accuracy of the calibration model. Sixty samples, which were not involved in the calibration, were tested using the calibration equation. Table 3 gives the external validation statistics of the near-infrared spectroscopy model. The correlation coefficient between the measured and predicted sample water content was 0.973 , which indicated that there was good correlation between the 
near-infrared predicted value and the chemically measured value. The RMSEP (4.037\%) was very close to the previous RMSEC $(4.249 \%)$, suggesting good reliability of the prediction equation. The ratio of the performance to deviation (RPD) of the prediction model was greater than 3 (4.317). The water contents predicted from the near-infrared spectroscopy model were in good agreement with the measured values (Figure 3). Therefore, the calibration model had good prediction accuracy and could be used for actual detection.

Table 3 External validation statistics of the calibration model

\begin{tabular}{cccccc}
\hline Test parameters & $R_{p}$ & Bias & Slope & $R M S E P / \%$ & $R P D$ \\
\hline Value & 0.973 & -0.178 & 1.004 & 4.037 & 4.317 \\
\hline
\end{tabular}

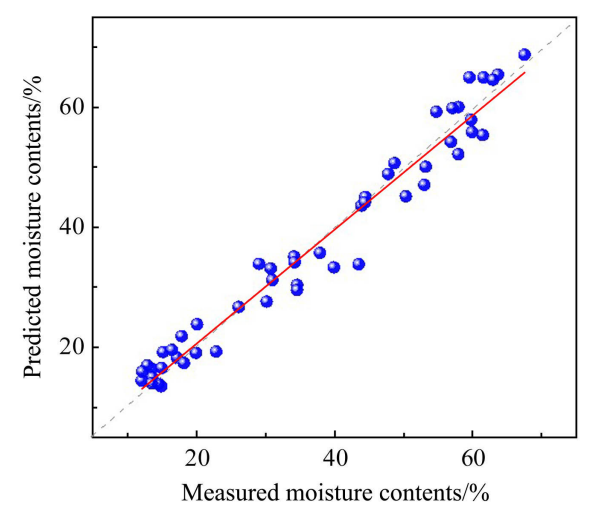

Figure 3 Correlations between measured and predicted values of corn stover silage

\subsection{Comparison of the near-infrared spectroscopy models of water content}

Table 4 lists the different near-infrared spectroscopy models of water content. In addition to straw ${ }^{[20,22]}$, the near-infrared spectroscopy models of water content have been established in fresh leaf ${ }^{[31]}$, seeds ${ }^{[9,29]}$, freeze-dried insulin ${ }^{[11]}$, and so on. According to the previous studies, the water change of sample is usually less than $20 \%$, while water content in this study ranged from $9.82 \%$ to $71.09 \%$. When referring to the optimal processing method, SNV and derivative are important processing method for spectral data in the establishment of water prediction model. All the optimal processing method includes one or both. The optimal processing method in this study was obtained by successive processing of MSC and 1-D. MSC could eliminate the spectral baseline shift due to the difference in size and shape of samples. From sample preparation, the difference in shape and size was found in corn stover silage, whereas not in other samples. In terms of research object, the Golden pothos leaf and corn stover silage were the most similar ${ }^{[31]}$. The RMSEC and correlation coefficient $(R)$ of water calibration model for Golden pothos leaf were $3.36 \%$ and 0.80 , while those for corn stove silage were $4.249 \%$ and 0.974 . The accuracy of the near-infrared model for water content of corn stover silage has been greatly improved. In terms of wide range of water change, the barley and corn stover silage were the most similar ${ }^{[29]}$. The RMSEP and correlation coefficient $\left(R_{p}\right)$ of water prediction model for barley were $5.36 \%$ and 0.90 , while those for corn stover silage were $4.037 \%$ and 0.973 The accuracy of the near-infrared model for water content of corn stover silage has been slightly improved.

Table 4 Comparison of the near-infrared spectroscopy models of water content

\begin{tabular}{|c|c|c|c|c|c|c|c|c|c|c|}
\hline \multirow{2}{*}{ Number } & \multirow{2}{*}{$\begin{array}{l}\text { Water } \\
\text { range/\% }\end{array}$} & \multirow{2}{*}{ Sample } & \multirow{2}{*}{$\begin{array}{l}\text { Optimal processing } \\
\text { method }\end{array}$} & \multicolumn{2}{|c|}{ Calibration set } & \multicolumn{2}{|c|}{ Validation set } & \multicolumn{2}{|c|}{ Prediction set } & \multirow{2}{*}{ Reference } \\
\hline & & & & $R M S E C / \%$ & $R$ & $R M S E C V / \%$ & $R_{C V}$ & $R M S E P / \%$ & $R_{P}$ & \\
\hline 1 & $5.13-20.44$ & Wheat/Rice straw & $\mathrm{SNV}+1-\mathrm{D}$ & - & - & 0.630 & - & 0.692 & 0.9331 & {$[20]$} \\
\hline 2 & $3.15-6.75$ & Corn stover & 1-D+Karl Norris & - & - & 0.547 & 0.861 & 1.325 & 0.9317 & {$[22]$} \\
\hline 3 & $6.92-13.71$ & Soybean & $1-\mathrm{D}+\mathrm{SNV}$ & 0.451 & 0.983 & 0.203 & 0.965 & - & 0.966 & [9] \\
\hline 4 & $28.5-97.9$ & Barley & $2-\mathrm{D}$ & - & - & 6.36 & 0.94 & 5.36 & 0.90 & [29] \\
\hline 6 & $32.8-61.7$ & Golden pothos leaf & SNV & 3.36 & 0.80 & 3.77 & 0.75 & - & - & {$[31]$} \\
\hline 7 & $9.82-71.09$ & Corn stover silage & $\mathrm{MSC}+1-\mathrm{D}$ & 4.249 & 0.974 & 4.256 & 0.949 & 4.037 & 0.973 & This study \\
\hline
\end{tabular}

Note: 1-D: the first-order derivation; 2-D: the second-order derivation; SNV: standard normal variate; MSC: multiplicative scatter correction.

$R M S E C$ : the root mean square error of calibration; RMSECV: the root mean square error of cross validation; RMSEP: the root mean square error of prediction

$R$ : the calibration correlation coefficient; $R_{C V}$, the cross validation correlation coefficient; $R_{P}$ the prediction correlation coefficient.

\section{Conclusions}

(1) In this study, corn stover silage samples were measured for water content and their corresponding near-infrared spectroscopy information were collected. The results show that the water content in the samples ranged from $9.82 \%$ to $71.09 \%$, and the different samples exhibited nearly similar spectral morphology. The absorption peak of the spectrum contained the multiplier and frequency spectrum band of water molecule vibration, which could provide the spectral information required for water detection.

(2) The calibration model for water content prediction was established by MPLS. In this experiment, derivation-based processing could effectively eliminate background-induced interference and baseline drift or rotation. The SNV and MSC preprocessing methods suggested that there was almost no optical path change in spectral response and the surface scattering characteristics of the sample had significant influence on the spectrum.

(3) The optimal calibration model for water content determination was successively preprocessed by MSC and 1-D after smoothing by four data interval window points (every $8 \mathrm{~nm}$ ). The correlation coefficients of the calibration and verification sets were 0.974 and 0.949 , respectively. The parameters of the calibration and verification sets corresponding to the calibration model were optimal.

(4) Sixty samples were externally tested via the calibration equation. The results showed that the correlation coefficient between the measured and predicted sample water content was 0.973 , suggesting that the calibration model had good prediction accuracy. It was concluded that near-infrared spectroscopy technology could be used for rapid and non-destructive testing of water content in corn stover silage. Further studies could explore the application of the near-infrared model for different varieties of corn stover silage. 


\section{Acknowledgements}

This work was supported by Commonwealth Project of Science and Technology Agency of Zhejiang Province, (No. 2017C32068, LGN18F030001); the Major Project of Zhejiang Science and Technology Department (2016C02G2100540), China.

\section{[References]}

[1] Zhao C, Shao Q, Li B, Ding W. Comparison of hydrogen peroxide and ammonia pretreatment of corn Stover: Solid Recovery, composition changes, and enzymatic hydrolysis. Energy \& Fuels, 2014; 28(10): 6392-6397.

[2] Qiao X, Zhao C, Shao Q, Hassan M. Structural characterization of corn stover lignin after hydrogen peroxide presoaking prior to ammonia fiber expansion pretreatment. Energy \& Fuels, 2018; 32(5): 6022-6030.

[3] Xu Z, He H, Zhang S, Kong J. Effects of inoculants Lactobacillus brevis and Lactobacillus parafarraginis on the fermentation characteristics and microbial communities of corn stover silage. Scientific Reports, 2017; 7(1): 13614 .

[4] Sun Y, Gong X, Wang Z, Huang C, Ma X, Wang M. Two-step pretreatment of corn stover silage using non-ionic surfactant and ferric nitrate for enhancing sugar recovery and enzymatic digestibility of cellulose. Applied Biochemistry Biotechnology, 2019; 189(1): 65-75.

[5] Sans S, Ferré J, Boqué R, Sabaté J, Casals J, Simó J. Determination of chemical properties in 'calçot' (Allium cepa L.) by near infrared spectroscopy and multivariate calibration. Food Chemistry, 2018; 262: $178-183$

[6] Li B, Long Y, Yang H. Measurements and analysis of water content in winter wheat leaf based on terahertz spectroscopy. Int J Agric \& Biol Eng, 2018; 11(3): 178-182.

[7] Büning-Pfaue H. Analysis of water in food by near infrared spectroscopy. Food Chemistry, 2003; 82(1):107-115.

[8] Czarnik-Matusewicz B, Pilorz S, Hawranek J P. Temperature-dependent water structural transitions examined by near-IR and mid-IR spectra analyzed by multivariate curve resolution and two-dimensional correlation spectroscopy. Analytica Chimica Acta, 2005; 544(1-2): 15-25.

[9] Zhu Z, Chen S, Wu X, Xing C, Yuan J. Determination of soybean routine quality parameters using near-infrared spectroscopy. Food Science \& Nutrition, 2018; 6(4): 1109-1118.

[10] Qin J, Kim M S, Chao K, Bellato L, Schmidt W F, Cho B, Huang M. Inspection of maleic anhydride in starch powder using line-scan hyperspectral Raman chemical imaging technique. Int J Agric \& Biol Eng, 2018; 11(6): 120-125.

[11] Grohganz H, Gildemyn D, Skibsted E, Flink J M, Rantanen J. Towards a robust water content determination of freeze-dried samples by near-infrared spectroscopy. Analytica Chimica Acta, 2010; 676(1-2): 34-40.

[12] Manley M, du Toit G, Geladi P. Tracking diffusion of conditioning water in single wheat kernels of different hardnesses by near infrared hyperspectral imaging. Analytica Chimica Acta, 2011; 686(1-2): 64-75.

[13] Zhao C, Qiao X, Cao Y, Shao Q. Application of hydrogen peroxide presoaking prior to ammonia fiber expansion pretreatment of energy crops. Fuel, 2017; 205(10): 184-191.

[14] Zhao C, Shao Q, Ma Z, Li B, Zhao X. Physical and chemical characterizations of corn stalk resulting from hydrogen peroxide presoaking prior to ammonia fiber expansion pretreatment. Industrial
Crops and Products, 2016; 83(5): 86-93.

[15] Bär D, Debus H, Brzenczek S, Fischer W, Imming P. Determining particle size and water content by near-infrared spectroscopy in the granulation of naproxen sodium. Journal of Pharmaceutical and Biomedical Analysis, 2018; 151(3): 209-218.

[16] Huang Y, Lu R, Chen K. Prediction of firmness parameters of tomatoes by portable visible and near-infrared spectroscopy. Journal of Food Engineering, 2018; 222(4): 185-198.

[17] Liu C, Han Y, Min S, Jia W, Meng X, Liu P. Rapid qualitative and quantitative analysis of methamphetamine, ketamine, heroin, and cocaine by near-infrared spectroscopy. Forensic Science International, 2018; 290(9): 162-168.

[18] Yang Y, Zhou S, Song J, Li G, Zhu S. Feasibility of terahertz spectroscopy for hybrid purity verification of rice seeds. Int J Agric \& Biol Eng, 2018; 11(5): 65-69.

[19] Zhang H, Zhu Q, Huang M, Guo Y. Automatic determination of optimal spectral peaks for classification of Chinese tea varieties using laser-induced breakdown spectroscopy. Int J Agric \& Biol Eng, 2018; 11(3): 154-158.

[20] Huang C, Han L, Liu X, Ma L. Rapid measurement for moisture and calorific value of straw based on near infrared spectroscopy and local algorithm. Journal of Infrared and Millimeter Wave, 2009; 28(3): 184-187.

[21] Huang C, Han L, Liu X, Yang Z. Proximate analysis of straw by near infrared spectroscopy (NIRS). Spectroscopy and Spectral Analysis, 2009; 29(04): 960-963. (in Chinese)

[22] Liu L, Chen H. Prediction of maize stover components with near infrared reflectance spectroscopy. Spectroscopy and Spectral Analysis, 2007; 27(02): 275-278. (in Chinese)

[23] Wu J, Bai Q, Su S, Chen S, Meng Q, Yan Y. Near-Infrared reflectance spectroscopy analysis of cellulose content in corn stalk. Chinese Journal of Analytical Chemistry, 2005; 33(10): 1421-1423. (in Chinese)

[24] Tai S, Zhang R, Shi J, Xue J, Zhang X, Ma G, Lu H. Prediction of forage quality of maize stover by near infrared spectroscopy. Transactions of the CSAE, 2009; 25(12): 151-155. (in Chinese)

[25] Bai Q, Chen S, Dong X, Meng Q, Yan Y, Dai J. Prediction of NDF and ADF concentrations with near infrared reflectance spectroscopy (NIRS). Spectroscopy and Spectral Analysis, 2004; 24(11): 1345-1347. (in Chinese)

[26] Wu J, Xi S, Zeng G, Wang D, Jiang Y, Wang M. Study on decomposed corn straw residue by Fourier transform infrared spectroscopy. Acta Pedologica Sinica, 1999; 34(1): 91-100. (in Chinese)

[27] GB/T 14095-2007. Drying technology of agricultural products-Terminology, 2007.

[28] GB/T 18868-2002. Method for determination of moisture, crude protein, crude fat, crude fibre, lysine and methinione in feeds-Near-infrared reflectance spectroscopy, 2002.

[29] Cozzolino D, Roumeliotis S, Eglinton J. Monitoring water uptake in whole barley (Hordeum vulgare L.) grain during steeping using near infrared reflectance spectroscopy. Journal of Food Engineering, 2013; 114(4): 545-549.

[30] Templeton D W, Sluiter A D, Hayward T K, Hames B R, Thomas S R. Assessing corn stover composition and sources of variability via NIRS. Cellulose, 2009; 16(4): 621-639.

[31] Higa S, Kobori H, Tsuchikawa S. Mapping of leaf water content using near-infrared hyperspectral imaging. Applied Spectroscopy, 2013; 67(11) 1302-1307. 